VOL. 44 (1991) [245-252]

\title{
A CLASS OF ONE-RELATOR GROUPS WITH CENTRE
}

\section{JAMES MCCOOL}

\begin{abstract}
Let the group $H$ have presentation $\left\langle a_{1}, \ldots, a_{m} ; a_{1}^{p_{1}}=a_{2}^{p_{1}}, \ldots, a_{m-1}^{p_{m-1}}=a_{m}^{p_{m-1}}\right\rangle$ where $m \geqslant 3, p_{i} \geqslant 2$ and $\left(p_{i}, p_{j}\right)=1$ if $i \neq j$. We show that $H$ is a onerelator group precisely if $H$ can be obtained from a suitable group $\left\langle a, b ; a^{p}=b^{p}\right\rangle$ by repeated applications of a (two-stage) procedure consisting of applying central Nielsen transformations followed by adjoining a root of a generator. We conjecture that any one-relator group $G$ with non-trivial centre and $G / G^{\prime}$ not free abelian of rank two can be obtained in the same way from a suitable group $\left\langle a, b ; a^{p}=b^{q}\right\rangle$.
\end{abstract}

\section{INTRODUCTION}

Let $G$ be a non-cyclic one-relator group with non-trivial centre and with commutator quotient group $G / G^{\prime}$ not free abelian of rank two. It was shown by Pietrowski [9] that $G$ has a presentation of the form

$$
\left\langle a_{1}, \ldots, a_{m} ; a_{1}^{p_{1}}=a_{2}^{q_{1}}, \ldots, a_{m-1}^{p_{m-1}}=a_{m}^{q_{m-1}}\right\rangle,
$$

where $m \geqslant 2, p_{i} \geqslant 2, q_{i} \geqslant 2$ and $\left(p_{i}, q_{j}\right)=1$ for $i>j$. This leads to the problem as to which groups $B$ satisfying these conditions actually have a one-relator presentation. In [7] Meskin, Pietrowski and Steinberg showed that this is always the case if $m=3$, and in general that any such group $H$ is generated by the pair $\left(a_{1}, a_{m}\right)$ and has centre generated by $a_{1}^{p_{1} \cdots p_{m-1}}=a_{m}^{q_{1} \cdots q_{m-1}}$; moreover, if $H$ is one-relator on $\left(a_{1}, a_{m}\right)$ and $p_{m} \equiv \pm 1$ modulo $q_{1} q_{2} \cdots q_{m-1}$, then

$$
\left\langle a_{1}, \cdots, a_{m+1} ; a_{1}^{p_{1}}=a_{2}^{q_{1}}, \cdots, a_{m}^{p_{m}}=a_{m+1}^{q_{m}}\right\rangle
$$

is one-relator on $\left(a_{1}, a_{m+1}\right)$ for any choice of $q_{m} \geqslant 2$. On the negative side, it was shown by Collins [3] that the group $\left\langle a_{1}, a_{2}, a_{3}, a_{4} ; a_{1}^{2}=a_{2}^{2}, a_{2}^{5}=a_{3}^{5}, a_{3}^{3}=a_{4}^{3}\right\rangle$ is not a one-relator group. Collins also showed in [3] that any generating set $(x, y)$ of a group of the form (1) is Nielsen equivalent to one of the form $\left(a_{1}^{r}, a_{m}^{s}\right)$, for suitable integers $r$ and $s$.

Received 3rd October, 1990.

Research supported by a grant from the Natural Sciences and Engineering Research Council of Canada. I would like to thank S. Krstić and E.C. Turner for stimulating conversation on the subject matter of this paper.

Copyright Clearance Centre, Inc. Serial-fee code: 0004-9729/91 \$A2.00+0.00. 
In the present paper we give a simple extension, in terms of Nielsen transformations, of the recipe of [7] mentioned above for constructing new one-relator groups of form (1) from known ones. Our main result is that this procedure gives, in particular, all one-relator groups of the form (1) which satisfy the additional conditions $p_{i}=$ $q_{i}(1 \leqslant i \leqslant m-1)$. This yields a very easy algorithm for testing whether or not a given presentation (1) with $p_{i}=q_{i}(1 \leqslant i \leqslant m-1)$ is a one relator group. Thus it turns out, for example, that the group $\left\langle a_{1}, a_{2}, a_{3}, a_{4} ; a_{1}^{2}=a_{2}^{2}, a_{2}^{p_{2}}=a_{3}^{p_{2}}, a_{3}^{3}=a_{4}^{3}\right\rangle$ is not a onerelator group for any choice of $p_{2} \geqslant 2$. This extends the example of Collins mentioned above.

\section{A Nielsen transformation procedure}

Let $\left(a_{1}^{r}, a_{m}^{\Delta}\right)$ be a generating set of a group $H$ of the form (1) above. Applying either of the Nielsen transformations

or

$$
\begin{aligned}
& \left(a_{1}^{r}, a_{m}^{s}\right) \longrightarrow\left(a_{1}^{r}, a_{m}^{\varepsilon s} a_{1}^{r k p_{1} p_{2} \cdots p_{m-1}}\right) \\
& \left(a_{1}^{r}, a_{m}^{*}\right) \longrightarrow\left(a_{1}^{e r} a_{m}^{e l q_{1} q_{2} \cdots q_{m-1}}, a_{m}^{s}\right)
\end{aligned}
$$

where $\varepsilon= \pm 1$ and $k, \ell$ are integers, will again yield such a generating set, since, for example, $a_{m}^{e s} a_{1}^{r k p_{1} p_{2} \cdots p_{m-1}}=a_{m}^{\varepsilon s+r k q_{1} q_{2} \cdots q_{m-1}}$. We shall call these central Nielsen transformations.

It is clear that if $(x, y)$ is a one-relator generating set of a group $G$, then so is any pair $\left(x_{1}, y_{1}\right)$ which is Nielsen equivalent to $(x, y)$. In particular, if $\left(a_{1}^{r}, a_{m}^{a}\right)$ is a onerelator generating set of a group $H$ of form (1), and $\left(a_{1}^{\lambda}, a_{m}^{\mu}\right)$ is Nielsen equivalent to this, then we have a presentation $\langle x, y ; R(x, y)=1\rangle$ (say) of $H$, with $x$ corresponding to $a_{1}^{\lambda}$ and $y$ to $a_{m}^{\mu}$. We now choose $q_{m} \geqslant 2$ and take $H_{1}$ to be the free product of $H$ with the infinite cycle on $a_{m+1}$, amalgamating $a_{m+1}^{q_{m}}=y^{\eta}$, where $\eta=1$ if $\mu>0$ and $\eta=-1$ if $\mu<0$. Now on the one hand $H_{1}$ is a one-relator group, since it has presentation $\left\langle x, a_{m+1} ; R\left(x, a_{m+1}^{q_{m}}\right)=1\right\rangle$, and on the other hand it has presentation

$$
\left\langle a_{1}, \ldots, a_{m+1} ; a_{1}^{p_{1}}=a_{2}^{q_{1}}, \ldots, a_{m}^{p_{m}}=a_{m+1}^{q_{m}}\right\rangle,
$$

where $p_{m}=|\mu|$, so that $H_{1}$ is of the form (1) if $p_{m} \geqslant 2$, while if $p_{m}=1$ this is again the case since then, by deleting the generator $a_{m}=a_{m+1}^{q_{m}}$, and renumbering $a_{m+1}$, (3) can be replaced by

$$
\left\langle a_{1}, \ldots, a_{m} ; a_{1}^{p_{1}}=a_{2}^{q_{1}}, \ldots, a_{r, 3-1}^{p_{m-1}}=a_{m-1}^{q_{m-1} q_{m}}\right\rangle .
$$

In either case the uniqueness result of Pietrowski [9], namely that the isomorphism class of a group of form (1) is uniquely determined by the sequence $p_{1}, q_{1}, \ldots, p_{m-1}, q_{m-1}$ 
together with its mirror image $q_{m-1}, p_{m-1}, \ldots, q_{1}, p_{1}$, shows that the group $H_{1}$ is not isomorphic to $H$. Furthermore, $H_{1}$ has a one-relator generating set of the same kind as the generating set $\left(a_{1}^{r}, a_{m}^{*}\right)$ of $H$, namely $\left(a_{1}^{\lambda}, a_{m+1}\right)$ if $H_{1}$ has presentation (3), and $\left(a_{1}^{\lambda}, a_{m}\right)$ if (4) applies. Of course the above process applies equally well if we adjoin a root of $a_{1}^{\lambda}$, rather than one of $a_{m}^{\mu}$.

Thus our procedure simply amounts to applying central Nielsen transformations to a one-relator generating set $\left(a_{1}^{r}, a_{m}^{*}\right)$ of a group $H$ of form (1), and then adjoining an $n$-th root $(n \geqslant 2)$ of one of the generators so obtained; the result is a group $H_{1}$ of the same type, with a corresponding one-relator generating set, and with $H_{1}$ not isomorphic to $H$. We note that the recipe of Meskin, Pietrowski and Steinberg [7] is the special case of this where the original one-relator generating set is just $\left(a_{1}, a_{m}\right)$, and a single central Nielsen transformation is applied before a root is adjoined.

As a simple illustration of the above, the group

$$
H=\left\langle a_{1}, a_{2}, a_{3}, a_{4} ; a_{1}^{p_{1}}=a_{2}^{3}, a_{2}^{2}=a_{3}^{5}, a_{3}^{13}=a_{4}^{q_{3}}\right\rangle
$$

is one-relator on the generating set $\left(a_{1}, a_{4}\right)$ for all choices of $p_{1} \geqslant 2, q_{3} \geqslant 2$. To see this we recall the observation of Zieschang [10] that if $p \geqslant 2$ and $q \geqslant 2$ then any generating set $\left(a^{r}, b^{s}\right)$ of $\left\langle a, b ; a^{p}=b^{q}\right\rangle$ with either $r=1$ or $s=1$ is a one-relator generating set. Now starting with the one-relator generating set $\left(a_{2}, a_{3}^{2}\right)$ of $H_{1}=\left\langle a_{2}, a_{3} ; a_{2}^{2}=a_{3}^{5}\right\rangle$, we apply Nielsen transformations as follows:

$$
\left(a_{2}, a_{3}^{2}\right) \longrightarrow\left(a_{2}^{-1} a_{3}^{10}, a_{3}^{2}\right)=\left(a_{2}^{3}, a_{3}^{2}\right) \longrightarrow\left(a_{2}^{3}, a_{2}^{6} a_{3}^{-2}\right)=\left(a_{2}^{3}, a_{3}^{13}\right)
$$

Thus $\left(a_{2}^{3}, a_{3}^{13}\right)$ is a one-relator generating set of $H_{1}$, and $H$ is obtained by adjoining roots $a_{1}, a_{4}$ of $a_{2}, a_{3}$ respectively, so that $H$ is one-relator on $\left(a_{1}, a_{4}\right)$ as claimed. We note that this example is not covered by Theorem 2 of [7], since 13 is not congruent to \pm 1 modulo 15 , and 3 is not congruent to \pm 1 modulo 26 .

We would conjecture that any one-relator group of the form (1) can be obtained by a number of applications of the above procedure, starting with a one-relator generating set $\left(a^{r}, b^{2}\right)$ of a suitable group $H_{1}=\left\langle a, b ; a^{p}=b^{q}\right\rangle$. It would in fact then be sufficient to start with such a set with either $r=1$ or $s=1$, since Collins [4] has shown that any one-relator generating set $\left(a^{r}, b^{a}\right)$ of $H_{1}$ is equivalent, by central Nielsen transformations, to one of this special form.

\section{The Main Result.}

In [3] Collins has shown that $\left(a_{1}^{r_{1}}, a_{m}^{*_{1}}\right)$ is a generating set of the group (1) if and only if $r_{1}$ and $s_{1}$ are non-zero integers satisfying $\left(r_{1}, s_{1}\right)=\left(r, p_{1} p_{2} \ldots p_{m-1}\right)=$ $\left(s, q_{1} q_{2} \ldots q_{m-1}\right)=1$. Moreover if $r_{1} \geqslant 1, s_{1} \geqslant 1$ and $2 r_{1}>s_{1} p_{1} p_{2} \ldots p_{m-1}$, then $r=$ 
$\left|r_{1}-s p_{1} p_{2} \ldots p_{m-1}\right|<r_{1}$, and $\left(a_{1}^{r}, a_{m}^{s_{1}}\right)$ is equivalent to $\left(a_{1}^{r_{1}}, a_{m}^{s_{1}}\right)$ by the central Nielsen transformation $\left(a_{1}^{r_{1}}, a_{m}^{\theta_{1}}\right) \longrightarrow\left(a_{1}^{\varepsilon r_{1}} a_{m}^{\varepsilon s_{1} q_{1} q_{2} \ldots q_{m-1}}, a_{m}^{s_{1}}\right)$, where $\varepsilon= \pm 1$ is chosen so that $r=\varepsilon\left(r_{1}-s_{1} q_{1} q_{2} \ldots q_{m-1}\right)$. A similar observation applies if $2 s_{1}>r_{1} q_{1} q_{2} \ldots q_{m-1}$. Thus it is clear that given a generating set $\left(a_{1}^{r_{1}}, a_{m}^{s_{1}}\right)$ of (1) we can find, algorithmically, a series of central Nielsen transformations that, when applied to $\left(a_{1}^{r_{1}}, a_{m}^{s_{1}}\right)$, yield a pair $\left(a_{1}^{r}, a_{m}^{s}\right)$ satisfying $r \geqslant 1, s \geqslant 1,2 r \leqslant s p_{1} p_{2} \ldots p_{m-1}$ and $2 s \leqslant r q_{1} q_{2} \ldots q_{m-1}$. We shall describe this by saying that $\left(a_{1}^{r_{1}}, a_{m}^{a_{1}}\right)$ centrally reduces to $\left(a_{1}^{r}, a_{m}^{*}\right)$, and that $\left(a_{1}^{r}, a_{m}^{*}\right)$ is centrally reduced. We can now state our main result as

THEOREM. Let the group $H$ have presentation

$$
\left\langle a_{1}, \ldots, a_{m} ; a_{1}^{p_{1}}=a_{2}^{p_{1}}, \ldots, a_{m-1}^{p_{m-1}}=a_{m}^{p_{m-1}}\right\rangle
$$

where $m \geqslant 3, p_{i} \geqslant 2$ and $\left(p_{i}, p_{j}\right)=1$ if $i \neq j$. If $H$ is a one-relator group, then $\left(a_{1}, a_{m}\right)$ is a one-relator generating set, and any one-relator generating set of the form $\left(a_{1}^{r}, a_{m}^{*}\right)$ centrally reduces to $\left(a_{1}, a_{m}\right)$. Moreover, the subgroup of $H$ generated by $\left(a_{2}, a_{m-1}\right)$ must be one-relator on the generating set $\left(a_{2}^{p_{1}}, a_{m-1}^{p_{m-1}}\right)$.

Proof: Suppose that $H$ is presented as above, and that $H$ is a one-relator group. Then by the results of Collins alluded to above we have a one-relator centrally reduced generating set $\left(a_{1}^{r}, a_{m}^{s}\right)$ of $H$, so that $r \geqslant 1, s \geqslant 1,2 r \leqslant s p, 2 s \leqslant r p$ where $p=$ $p_{1} p_{2} \ldots p_{m-1}$, and $(r, s)=(r, p)=(s, p)=1$. Now let $G=\langle x, y ; R(x, y)=1\rangle$ be isomorphic to $H$ by an isomorphism $\phi: G \rightarrow H$ such that $\phi(x)=a_{1}^{r}$ and $\phi(y)=$ $a_{m}^{*}$. We may assume $R(x, y)$ is of the form $R(x, y)=x^{m_{1}} y^{n_{1}} \ldots x^{m_{t}} y^{n_{t}}$ for non-zero integers $m_{i}, n_{i}(1 \leqslant i \leqslant t)$. Here we will have $t \geqslant 2$, since $m \geqslant 3$. We then have $a_{1}^{r m_{1}} a_{m}^{s n_{1}} \ldots a_{1}^{r m_{t}} a_{m}^{s n_{t}}=1$ in $H$, and by Corollary 2.3 of [3] it follows that either $p_{1} \mid m_{i}$ and $p_{m-1} \mid n_{i}$ for $1 \leqslant i \leqslant t$, or $p$ divides some $m_{i}$ or $n_{j}$.

We suppose firstly that $m_{i}=p_{1} m_{i}^{\prime}$ and $n_{i}=p_{m-1} n_{i}^{\prime}$ for $1 \leqslant i \leqslant t$. We then have

$$
G=\left\langle x, y ; x^{p_{1} m_{1}^{\prime}} y^{p_{m-1} n_{1}^{\prime}} \ldots x^{p_{1} m_{z}^{\prime}} y^{p_{m-1} n_{t}^{\prime}}=1\right\rangle
$$

so that, if $G_{1}$ is given by

$$
G_{1}=\left\langle\alpha, \beta ; \alpha^{m_{1}^{\prime}} \beta^{n_{1}^{\prime}} \ldots \alpha^{m_{i}^{\prime}} \beta^{n_{t}^{\prime}}=1\right\rangle,
$$

then $G$ can be described as the (repeated) free product with amalgamation

$$
G=\langle x\rangle_{x^{p_{1}^{*}=\alpha}}^{\star} \quad G_{1} \underset{\beta=y^{p_{m-1}}}{\star}\langle y\rangle .
$$

Now $\phi\left(x^{p_{1}}\right)=a_{1}^{r p_{1}}=a_{2}^{r p_{1}}$ and $\phi\left(y^{p_{m-1}}\right)=a_{m}^{s p_{m-1}}=a_{m-1}^{s p_{m-1}}$, so that $\phi$ induces an isomorphism from $G_{1}$ to the subgroup of $H$ generated by $\left(a_{2}^{r p_{1}}, a_{m-1}^{s p_{m-1}}\right)$. Now this 
subgroup is just the subgroup $\left\langle a_{2}, a_{m-1}\right\rangle$ generated by $\left(a_{2}, a_{m-1}\right)$, and it follows that $\left\langle a_{2}, a_{m-1}\right\rangle$ is one-relator on the generating set $\left(a_{2}^{r p_{1}}, a_{m-1}^{s p_{m-1}}\right)$. However $\left\langle a_{2}, a_{m-1}\right\rangle$ also has presentation

$$
\left\langle a_{2}, \ldots, a_{m-1} ; a_{2}^{p_{2}}=a_{3}^{p_{2}}, \ldots, a_{m-2}^{p_{m-2}}=a_{m-1}^{p_{m-2}}\right\rangle
$$

(in case $m=3$ this is just the infinite cycle on $a_{2}$ ). Thus $G_{1}$ is isomorphic to the group given by (6), by an isomorphism taking $\alpha$ to $a_{2}^{r p_{1}}$ and $\beta$ to $a_{m-1}^{s p_{m-1}}$. It follows from the description of $G$ as a free product with amalgamation that $G$ has presentation

$$
\left\langle x, a_{1}, \ldots, a_{m_{1}} y ; x^{p_{1}}=a_{2}^{r p_{1}}, a_{2}^{p_{2}}=a_{3}^{p_{2}}, \ldots, a_{m-2}^{p_{m-2}}=a_{m-1}^{p_{m-2}}, a_{m-1}^{* p_{m-1}}=y^{p_{m-1}}\right\rangle
$$

This contradicts the uniqueness result of [9] unless $r=s=1$. This proves the theorem in the case under consideration.

We may now suppose that $p$ divides some $m_{i}$ or $n_{j}$. We will show that this is in fact impossible. Our arguments here use a number of standard results as developed, for example, in Murasugi [8], Baumslag and Taylor [1] and Collins [4]; the basic technique of course is due to Magnus (see Magnus, Karrass and Solitar [6]). We may suppose, without loss of generality, that $m_{1}>0$ and $p \mid m_{1}, m_{1}=p m_{1}^{\prime}$ say. We now define the group $J$ by

$$
J=\left\langle e, a_{1}, \ldots a_{m}, d ; e^{r}=a_{1}^{r}, a_{1}^{p_{1}}=a_{2}^{p_{1}}, \ldots, a_{m-1}^{p_{m-1}}=a_{m}^{p_{m-1}}, a_{m}^{s}=d^{z}\right\rangle
$$

and we clearly have $J=\left\langle e, d ; R\left(e^{r}, d^{*}\right)=1\right\rangle$. Now $e^{r s p}=d^{r s p}$ in $J$, so that the exponent sums of $e$ and $d$ in $R\left(e^{r}, d^{*}\right)$ are equal in magnitude and opposite in sign. Introducing a new generator $z=e d^{-1}$ and eliminating $e=z d$, we obtain $J=\left\langle z, d ; R\left((z d)^{r}, d^{s}\right)=1\right\rangle$, where

$$
R\left((z d)^{r}, d^{*}\right) \equiv(z d)^{r p m_{1}^{\prime}} d^{s n_{1}}(z d)^{r m_{2}} d^{s n_{2}} \ldots(z d)^{r m_{t}} d^{e n_{t}}
$$

has exponent sum zero on $d$. It is well known in this context (see for example, [1]) that $J$ is an extension of a free group $F$ of finite rank, by the infinite cycle on $d$. Here $F$ is generated by the conjugates $z_{i}=d^{i} z d^{-i}$ of $z$ by powers of $d$, and if $R\left((z d)^{r}, d^{\prime}\right)$ is rewritten in terms of the $z_{i}$, as $R_{0}=z_{0} z_{1} \ldots z_{r p m_{1}^{\prime}-1} w$ say, where $w$ is the rewritten form of $d^{r p m_{1}^{\prime}+e n_{1}}(z d)^{r m 2} d^{s n_{2}} \ldots(z d)^{r m_{t}} d^{s n_{t}}$, then $z_{0} z_{1} \ldots z_{r p m_{1}^{\prime}-1}$ is a subword of $R_{0}$. Moreover if $\lambda, \mu$ are, respectively, the least and greatest subscripts on $z$ occurring in $R_{0}$, then $F$ has rank $r(F)$ given by $r(F)=\mu-\lambda$.

It follows immediately from the above observations that $r(F) \geqslant r p m_{1}^{\prime}-1 \geqslant r p-1$. Now the rank of $F$ can also be obtained from the presentation (7) of $J$ by an Euler 
characteristic argument: see Karrass, Pietrowski and Solitar [5] and Proposition 7.3 of Chapter IX of Brown [2]. We obtain from this

$$
r(F)=r+s+\left(\sum_{i=1}^{m-1} p_{i}\right)-m-1,
$$

so that

$$
r+s+\sum_{i=1}^{m-1} p_{i} \geqslant r p+m
$$

We will show that (8) is impossible. First we note that $2 s \leqslant r p-1$ (since $2 s=r p$ implies $s=1$ and so $r=1$ and $2=p$, which is impossible since $m \geqslant 3$ implies $\left.p=p_{1} p_{2} \ldots p_{m-1} \geqslant 6\right)$. We have therefore, from (8),

$$
r+\sum_{i=1}^{m-1} p_{i} \geqslant \frac{1}{2} r p=\frac{1}{2}(2 m+1) .
$$

When $m=3$ and $r=1$, (9) becomes $p_{1}+p_{2} \geqslant p_{1} p_{2} / 2+5 / 2$, so that $p_{1} p_{2}-2 p_{1}-$ $2 p_{2}+5 \leqslant 0$, or $\left(p_{1}-2\right)\left(p_{2}-2\right)+1 \leqslant 0$, which is impossible. We now use induction on $r$ to establish that (9) is false for $m=3$. Accordingly, we take the least $r>1$ so that

$$
r+p_{1}+p_{2} \geqslant \frac{r p_{1} p_{2}}{2}+\frac{7}{2}
$$

We have then

$$
r-1+p_{1}+p_{2}<(r-1) \frac{p_{1} p_{2}}{2}+\frac{7}{2}
$$

and combining these yields $2 \geqslant p_{1} p_{2}$, which is incorrect. Thus (9) is false for $m=3$ and all $r$. We now use induction on $m$ to show (9) is always false. Take $m \geqslant 4$ least so that (9) holds. We then have

$$
r+\sum_{i=1}^{m-2} p_{i}<\frac{r p_{1} p_{2} \ldots p_{m-2}}{2}+\frac{2 m-1}{2}
$$

We claim

$$
p_{m-1} \leqslant\left(p_{m-1}-1\right) r \frac{p_{1} p_{2} \cdots p_{m-2}}{2}+1
$$


Indeed if we put $\lambda=p_{m-1}$ and $\mu=r p_{1} p_{2} \cdots p_{m-2}$ then (11) is just $(\lambda-1) \mu / 2-\lambda+1 \geqslant$ 0 , that is, $\lambda \mu-\mu-2 \lambda+2 \geqslant 0$, or $(\lambda-2)(\mu-2)+\mu-2 \geqslant 0$, which is the case since $\lambda \geqslant 2$ and $\mu \geqslant 2$. Now adding (10) and (11) shows that (9) is false. This contradicts the existence of $m$ such that (9) is true. Hence (9), and therefore (8), is always false. This proves the theorem.

As an easy consequence, we have the

COROLLARY. There is an algorithm to decide, given a presentation (5), whether or not the corresponding group is a one-relator group.

Proof: Let the group $H=H_{m}$ be given by (5). According to the theorem, if $H$ is one-relator then the group $H_{m-2}=\left\langle a_{2}, a_{m-1}\right\rangle$ must be one-relator on the generating set $\left(a_{2}^{p_{1}}, a_{m-1}^{p_{m-1}}\right)$; conversely, if this is the case then clearly $H$ is one-relator (on $\left.\left(a_{1}, a_{m}\right)\right)$. So the question reduces to deciding if $H_{m-2}$ is one-relator on $\left(a_{2}^{p_{1}}, a_{m-1}^{p_{m-1}}\right)$. If $m=3$ this is the case (and of course we have a special case of a result of [7] noted previously), while if $m=4$ we may apply central Nielsen transformations to the generating set $\left(a_{2}^{p_{1}}, a_{3}^{p_{3}}\right)$ of $H_{2}=\left\langle a_{2}, a_{3} ; a_{2}^{p_{2}}=a_{3}^{p_{2}}\right\rangle$ to obtain a Nielsen equivalent centrally reduced set $\left(a_{2}^{r}, a_{3}^{s}\right)$, and the theorem of [4] tells us that $H_{2}$ is one-relator on $\left(a_{2}^{p_{1}}, a_{3}^{p_{3}}\right)$ precisely if $r=1$ or $s=1$ holds. Thus we can decide the question in this case. If $m>4$ then the theorem applies to $H_{m-2}$. Thus we centrally reduce $\left(a_{2}^{p_{1}}, a_{m-1}^{p_{m-1}}\right)$. If we do not obtain $\left(a_{2}, a_{m-1}\right)$ then $H$ is not one-relator. If we do obtain $\left(a_{2}, a_{m-1}\right)$, then $H$ is one-relator precisely if the same is true for $H_{m-2}$. This proves the result.

We now consider the group $H=\left\langle a_{1}, a_{2}, a_{3}, a_{4} ; a_{1}^{2}=a_{2}^{2}, a_{2}^{p_{2}}=a_{3}^{p_{2}}, a_{3}^{3}=a_{4}^{3}\right\rangle$, with $p_{2} \geqslant 2$. If $\left(2, p_{2}\right) \neq 1$ or $\left(3, p_{2}\right) \neq 1$ then it follows from [7] that $H$ is not one-relator, so we suppose $\left(2, p_{2}\right)=\left(3, p_{2}\right)=1$. Now we know $H$ is one-relator if, and only if, $\left(a_{2}^{2}, a_{3}^{3}\right)$ is a one-relator generating set of $H_{1}=\left\langle a_{2}, a_{3} ; a_{2}^{p_{2}}=a_{3}^{p_{2}}\right\rangle$. Now it is clear that $\left(a_{2}^{2}, a_{3}^{3}\right)$ is a centrally reduced generating set of $H_{1}$ (since $p_{2} \geqslant 5$ ), and it follows that it is not a one-relator generating set of $H_{1}$. Hence $H$ is not a one-relator group.

\section{REFERENCES}

[1] G. Baumslag and T. Taylor, 'The center of groups with one defining relator', Math. Ann. 175 (1968), 315-319.

[2] K.S. Brown, Cohomology of groups (Springer-Verlag, Berlin, Heidelberg, New York, 1982).

[3] D.J. Collins, 'Generation and presentation of one-relator groups with centre', Math. Z. 157 (1978), 63-77.

[4] D.J. Collins, 'Presentations of the amalgamated free product of two infinite cycles', Math. Ann. 237 (1978), 233-241.

[5] A. Karrass, A. Pietrowski and D. Solitar, 'Finite and infinite cyclic extensions of free groups', J. Austral. Math. Soc. 16 (1973), 458-466. 
[6] W. Magnus, A. Karrass, and D. Solitar, Combinatorial group theory (Interscience, New York, 1966).

[7] S. Meskin, A. Pietrowski and A. Steinberg, 'One-relator groups with center', J. Austral. Math. Soc. 16 (1973), 319-323.

[8] K. Murasugi, 'The center of a group with a single defining relation', Math. Ann. 155 (1964), 246-251.

[9] A. Pietrowski, 'The isomorphism problem for one-relator groups with non-trivial centre', Math. Z. 136 (1974), 95-106.

[10] H. Zieschang, 'Generators of the free product with amalgamation of two infinite cyclic groups', Math. Ann. 227 (1977), 195-221.

Department of Mathematics

University of Toronto

Toronto, Ontario

Canada M5S 1A1 